\title{
A comparison of finite difference and finite volume methods for solving the space-fractional advection-dispersion equation with variable coefficients \\ H. Hejazi ${ }^{1} \quad$ T. Moroney ${ }^{2} \quad$ F. Liu ${ }^{3}$
}

(Received 1 November 2012; revised 13 August 2013)

\begin{abstract}
Transport processes within heterogeneous media may exhibit nonclassical diffusion or dispersion which is not adequately described by the classical theory of Brownian motion and Fick's law. We consider a space-fractional advection-dispersion equation based on a fractional Fick's law. Zhang et al. [Water Resour. Res. 43:W05439, 2007] considered such an equation with variable coefficients, which they discretised using the finite difference method proposed by Meerschaert and Tadjeran [J. Comput. and Appl. Math. 172:65-77, 2004]. For this method, the presence of variable coefficients necessitates applying
\end{abstract}

http://journal.austms.org.au/ojs/index.php/ANZIAMJ/article/view/6333 gives this article, (C) Austral. Mathematical Soc. 2013. Published September 13, 2013, as part of the Proceedings of the 16th Biennial Computational Techniques and Applications Conference. ISSN 1446-8735. (Print two pages per sheet of paper.) Copies of this article must not be made otherwise available on the internet; instead link directly to this URL for this article. 
the product rule before discretising the Riemann-Liouville fractional derivatives using standard or shifted Grünwald formulas, depending on the fractional order. As an alternative, we propose using a finite volume method that deals directly with the equation in conservative form. Fractionally shifted Grünwald formulas are used to discretise the Riemann-Liouville fractional derivatives at control volume faces, eliminating the need for product rule expansions. We compare the two methods for several case studies, highlighting the convenience of the finite volume approach.

\section{Contents}

1 Introduction

C558

2 Numerical methods

C560

2.1 Finite difference method . . . . . . . . . . . . . . C560

2.2 Finite volume method . . . . . . . . . . . . . C563

3 Numerical experiments

C566

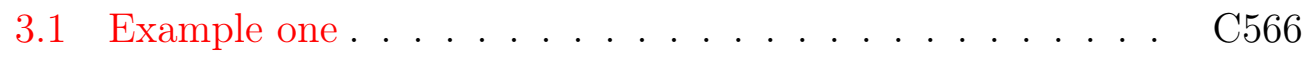

3.2 Example two . . . . . . . . . . . . . . . . . . C569

4 Conclusion

C571

References

C571

\section{Introduction}

Transport processes within complex and nonhomogeneous media may exhibit non-classical diffusion or dispersion which is not adequately described by the classical theory of Brownian motion and Fick's law $[1,2,3,12,13]$. 
Fractional differential equations, and fractional calculus in general, provide a means for modelling such anomalous transport by replacing traditional integer order derivatives with fractional derivatives. The application to anomalous transport is a significant driving force behind the rapid growth and expansion of the literature in the field of fractional calculus.

As far as numerical methods for solving fractional differential equations are concerned, finite difference methods were amongst the first developed. Meerschaert and Tadjeran [6, 7], and Tadjeran et al. [10] published several key papers in which they derived finite difference methods for equations involving Riemann-Liouville fractional derivatives. They showed that for fractional orders between zero and one, standard Grünwald formulas lead to stable methods, whereas for fractional orders between one and two, shifted Grünwald formulas are required for stability.

More recently, finite volume methods, which deal directly with equations in conservative form, were proposed. A finite volume method for solving the space-fractional advection-dispersion equation with constant coefficients was proposed by Zhang et al. [11]. Their method was based on discretising the integral using the Riemann-Liouville definition of the fractional derivative.

Previously we proposed a finite volume method for the two sided spacefractional advection-dispersion equation with constant coefficients [5]. Our method used shifted Grünwald formulas to discretise the fractional derivatives at control volume faces. We also proved the stability and convergence of the method.

The finite difference method of Meerschaert and Tadjeran [6] and the finite volume method of Hejazi et al. [5] both employ Grünwald formulas in their discretisations. A key difference between the two methods is that the finite volume method deals directly with the differential equation in conservative form, eliminating the need for product rule expansions in variable coefficient problems.

We consider the space-fractional advection-dispersion equation with variable 
coefficients,

$$
\frac{\partial C(x, t)}{\partial t}+\frac{\partial}{\partial x}[V(x) C(x, t)]=\frac{\partial}{\partial x}\left[K(x) \frac{\partial^{\alpha} C(x, t)}{\partial x^{\alpha}}\right],
$$

on the interval $x \in[a, b]$, subject to homogeneous Dirichlet boundary conditions. The function $C(x, t)$ represents, for example, a concentration, and $\mathrm{V}(\mathrm{x})$ and $\mathrm{K}(\mathrm{x})$ are the velocity and the anomalous dispersion coefficients, respectively. The operator $\partial^{\alpha} / \partial x^{\alpha}$ is the left Riemann-Liouville fractional derivative of order $\alpha[9$, p.62] and we assume $\alpha \in(0,1)$.

We derive the finite difference and finite volume discretisations for equation (1) and compare the numerical solution obtained with the two methods for several variable coefficient test problems. We demonstrate that the finite volume method produces solutions that conserve mass, whereas precise mass conservation is not achieved using the finite difference method.

\section{Numerical methods}

\section{$2.1 \quad$ Finite difference method}

We consider a transport domain $[a, b]$ that is discretised with $N+1$ uniformly spaced nodes $x_{i}=a+i h$ for $i=0, \ldots, N$, with the spatial step $h=(b-a) / N$. To numerically solve the space-fractional advection-dispersion equation using the finite difference method of Meerschaert and Tadjeran [6], we first expand the spatial derivative terms in (1) using the product rule. For the advective term,

$$
\frac{\partial}{\partial x}[V(x) C(x, t)]=\frac{\partial V(x)}{\partial x} C(x, t)+V(x) \frac{\partial C(x, t)}{\partial x},
$$

and for the dispersive term,

$$
\frac{\partial}{\partial x}\left[K(x) \frac{\partial^{\alpha} C(x, t)}{\partial x^{\alpha}}\right]=\frac{\partial K(x)}{\partial x} \frac{\partial^{\alpha} C(x, t)}{\partial x^{\alpha}}+K(x) \frac{\partial^{\alpha+1} C(x, t)}{\partial x^{\alpha+1}} .
$$


The derivative $\partial C / \partial x$ in (2) is approximated using second order central differences. This implies that a suitably fine mesh be used to ensure monotonicity [8]. We approximate the $\alpha$ and $\alpha+1$ order fractional derivatives with standard and shifted Grünwald formulas, respectively [6].

Definition 1. Shifted Grünwald formula on $[\mathrm{a}, \mathrm{b}]$

$$
\frac{\partial^{\alpha} \mathrm{C}(x, \mathrm{t})}{\partial x^{\alpha}} \approx \frac{1}{h^{\alpha}} \sum_{j=0}^{[(x-a) / h+p]}(-1)^{j}\left(\begin{array}{l}
\alpha \\
j
\end{array}\right) C[x-(j-p) h, t],
$$

where $\mathrm{p}$ is the shift value.

We define weights

$$
w_{0}^{\alpha}=1 \quad \text { and } \quad w_{\alpha, j}=(-1)^{j} \frac{\alpha(\alpha-1) \cdots(\alpha-j+1)}{j !} \text { for } j=1,2, \ldots,
$$

and write (4) more simply as

$$
\frac{\partial^{\alpha} \mathrm{C}(x, \mathrm{t})}{\partial x^{\alpha}} \approx \frac{1}{h^{\alpha}} \sum_{j=0}^{[(x-a) / h+p]} w_{j}^{\alpha} \mathrm{C}[x-(j-p) h, t] .
$$

Equation (6) with $p=0$ is the standard Grünwald formula. The shift value $p=1$ is required in the discretisation of the $\alpha+1$ order fractional derivative (recalling that $0<\alpha<1$ ) so that the resulting finite difference method is numerically stable [6]. Hence we arrive at the discretisations [6]

$$
\begin{aligned}
\frac{\partial^{\alpha} \mathrm{C}\left(x_{i}, \mathrm{t}\right)}{\partial x^{\alpha}} & \approx \frac{1}{h^{\alpha}} \sum_{j=0}^{i} w_{j}^{\alpha} \mathrm{C}\left(x_{i-j}, t\right), \\
\frac{\partial^{\alpha+1} \mathrm{C}\left(x_{i}, \mathrm{t}\right)}{\partial x^{\alpha+1}} & \approx \frac{1}{h^{\alpha+1}} \sum_{j=0}^{i+1} w_{j}^{\alpha+1} \mathrm{C}\left(x_{i-j+1}, \mathrm{t}\right) .
\end{aligned}
$$


We now define a temporal partition $t_{n}=n \tau$ for $n=0,1, \ldots$, where $\tau$ is the time step, and approximate the temporal derivative in (1) by the standard first order backward difference. Defining $\mathrm{C}_{i}^{n} \approx \mathrm{C}\left(\mathrm{x}_{i}, \mathrm{t}_{\mathrm{n}}\right)$ as the numerical solution, and using the spatial discretisations just derived, we obtain the fully implicit scheme [12]

$$
\begin{aligned}
\frac{C_{i}^{n+1}-C_{i}^{n}}{\tau}= & -V_{i}^{\prime} C_{i}^{n+1}-\frac{V_{i}}{2 h}\left[C_{i+1}^{n+1}-C_{i-1}^{n+1}\right] \\
& +K_{i}^{\prime}\left[\frac{1}{h^{\alpha}} \sum_{j=0}^{i} w_{j}^{\alpha} C_{i-j}^{n+1}\right]+K_{i}\left[\frac{1}{h^{\alpha+1}} \sum_{j=0}^{i+1} w_{j}^{\alpha+1} C_{i-j+1}^{n+1}\right],
\end{aligned}
$$

where $V_{i}^{\prime}=\partial V\left(x_{i}\right) / \partial x$ and $K_{i}^{\prime}=\partial K\left(x_{i}\right) / \partial x$. On collecting like terms the scheme becomes

$$
\frac{C_{i}^{n+1}-C_{i}^{n}}{\tau}=\sum_{j=0}^{N} f_{i j} C_{j}^{n+1}+\sum_{j=0}^{N} g_{i j} C_{j}^{n+1},
$$

with coefficients

$$
f_{i j}= \begin{cases}h^{-\alpha} K_{i}^{\prime} w_{i-j}^{\alpha} & \text { for } j<i-1 \\ h^{-\alpha} K_{i}^{\prime} w_{1}^{\alpha} & \text { for } j=i-1 \\ h^{-\alpha} K_{i}^{\prime} w_{0}^{\alpha}-V_{i}^{\prime} & \text { for } j=i \\ 0 & \text { for } j \geqslant i+1\end{cases}
$$

and

$$
g_{i j}= \begin{cases}h^{-(\alpha+1)} K_{i} w_{i-j+1}^{\alpha+1} & \text { for } j<i-1, \\ h^{-(\alpha+1)} K_{i} w_{2}^{\alpha+1}+V_{i} /(2 h) & \text { for } j=i-1, \\ h^{-(\alpha+1)} K_{i} w_{1}^{\alpha+1} & \text { for } j=i \\ h^{-(\alpha+1)} K_{i} w_{0}^{\alpha+1}-V_{i} /(2 h) & \text { for } j=i+1, \\ 0, & \text { for } j>i+1 .\end{cases}
$$

Defining the numerical solution vector $\mathbf{C}^{\mathfrak{n}}=\left(\mathrm{C}_{1}^{\mathfrak{n}}, \ldots, \mathrm{C}_{\mathrm{N}-1}^{\mathfrak{n}}\right)$, we solve the vector equation

$$
(I+\tau A+\tau B) C^{n+1}=C^{n},
$$


at each time step, where the matrices $A$ and $B$ have elements $A_{i j}=-f_{i j}$ and $B_{i j}=-g_{i j}$, respectively.

\subsection{Finite volume method}

Comparing (1) with the general transport equation

$$
\frac{\partial C(x, t)}{\partial t}=-\frac{\partial Q(x, t)}{\partial x}
$$

we identify the total flux

$$
\mathrm{Q}(x, \mathrm{t})=\mathrm{V}(\mathrm{x}) \mathrm{C}(x, \mathrm{t})+\mathrm{q}(x, \mathrm{t})
$$

with advective component

$$
V(x) C(x, t)
$$

and dispersive component

$$
q(x, t)=-K(x) \frac{\partial^{\alpha} C(x, t)}{\partial x^{\alpha}} .
$$

We again consider a transport domain $[a, b]$ that is discretised with $\mathrm{N}+1$ uniformly spaced nodes $x_{i}=a+i h$ for $i=0 \ldots N$, with the spatial step $\mathrm{h}=(\mathrm{b}-\mathrm{a}) / \mathrm{N}$. A finite volume discretisation is applied by integrating (14) over the ith control volume $\left[x_{i-1 / 2}, x_{i+1 / 2}\right]$,

$$
\int_{x_{i-1 / 2}}^{x_{i+1 / 2}} \frac{\partial C(x, t)}{\partial t} d x=-\int_{x_{i-1 / 2}}^{x_{i+1 / 2}} \frac{\partial Q(x, t)}{\partial x} d x .
$$

After interchanging the order of integration and differentiation on the left, and performing the integration on the right,

$$
\frac{d}{d t} \int_{x_{i-1 / 2}}^{x_{i+1 / 2}} C(x, t) d x=-\left[Q\left(x_{i+1 / 2}, t\right)-Q\left(x_{i-1 / 2}, t\right)\right],
$$


This leads to the standard finite volume discretisation

$$
\frac{\mathrm{d} \overline{\mathrm{C}}_{\mathrm{i}}}{\mathrm{dt}}=\frac{1}{\mathrm{~h}}\left[\left.\mathrm{Q}\right|_{x_{\mathrm{i}-1 / 2}}-\left.\mathrm{Q}\right|_{x_{i+1 / 2}}\right]
$$

where $\bar{C}_{i}$ is the control volume average $\bar{C}_{i}=\frac{1}{h} \int_{x_{i-1 / 2}}^{x_{i+1 / 2}} \mathrm{C} d x$. No approximations have been introduced at this point.

The flux $\mathrm{Q}$ has both advective and dispersive components. The key feature of our finite volume method is the approximation of the dispersive flux $\left.q\right|_{x_{i \pm 1 / 2}}$ by fractionally shifted Grünwald formulas [5]. The fractional shift $p=1 / 2$ in (6) allows us to build approximations of fractional derivatives at control volume faces $x_{i \pm 1 / 2}$ in terms of function values at the nodes $x_{j}$. This leads to the fractionally shifted Grünwald formulas

$$
\frac{\partial^{\alpha} \mathrm{C}\left(\mathrm{x}_{i-1 / 2}, \mathrm{t}\right)}{\partial x^{\alpha}} \approx \frac{1}{h^{\alpha}} \sum_{j=0}^{i} w_{j}^{\alpha} \mathrm{C}\left(x_{i-j}, \mathrm{t}\right),
$$

at the face $x_{i-1 / 2}$, and

$$
\frac{\partial^{\alpha} \mathrm{C}\left(x_{i+1 / 2}, t\right)}{\partial x^{\alpha}} \approx \frac{1}{h^{\alpha}} \sum_{j=0}^{i+1} w_{j}^{\alpha} C\left(x_{i-j+1}, t\right),
$$

at the face $x_{i+1 / 2}$.

The dispersive flux is approximated at the face $\boldsymbol{x}_{i-1 / 2}$ by

$$
\mathrm{q}\left(\mathrm{x}_{\mathrm{i}-1 / 2}, \mathrm{t}\right) \approx-\mathrm{K}\left(\mathrm{x}_{\mathrm{i}-1 / 2}\right)\left[\frac{1}{\mathrm{~h}^{\alpha}} \sum_{j=0}^{i} w_{j}^{\alpha} \mathrm{C}\left(\mathrm{x}_{\mathrm{i}-\mathrm{j}}, \mathrm{t}\right)\right],
$$

and at the face $x_{i+1 / 2}$ by

$$
\mathrm{q}\left(\mathrm{x}_{\mathrm{i}+1 / 2}, \mathrm{t}\right) \approx-\mathrm{K}\left(\mathrm{x}_{\mathrm{i}+1 / 2}\right)\left[\frac{1}{\mathrm{~h}^{\alpha}} \sum_{j=0}^{i+1} w_{j}^{\alpha} \mathrm{C}\left(\mathrm{x}_{\mathrm{i}-\mathrm{j}+1}, \mathrm{t}\right)\right] .
$$


Previously [5] we showed this discretisation to be of first order spatial accuracy for the constant coefficient case. In Section 3 we show numerically that the method retains first order spatial accuracy for the variable coefficient test problem.

For the advective flux $\mathrm{V}(\mathrm{x}) \mathrm{C}(\mathrm{x}, \mathrm{t})$ we use a standard averaging scheme

$$
\mathrm{V}\left(x_{i \pm 1 / 2}\right) \mathrm{C}\left(x_{i \pm 1 / 2}, t\right) \approx \frac{V\left(x_{i \pm 1 / 2}\right)}{2}\left[C\left(x_{i}, t\right)+C\left(x_{i \pm 1}, t\right)\right]
$$

which completes the spatial discretisation.

We now define a temporal partition $t_{n}=n \tau$ for $n=0,1, \ldots$, where $\tau$ is the time step, and approximate the temporal derivative in (20) by the standard first order backward difference. Defining $\mathrm{C}_{i}^{n} \approx \mathrm{C}\left(\mathrm{x}_{i}, \mathrm{t}_{\mathrm{n}}\right)$ as the numerical solution, and using the spatial discretisations just derived, we obtain the fully implicit scheme

$$
\begin{aligned}
\frac{C_{i}^{n+1}-C_{i}^{n}}{\tau}= & \frac{V_{i-1 / 2}}{2 h}\left[C_{i}^{n+1}+C_{i-1}^{n+1}\right]-\frac{V_{i+1 / 2}}{2 h}\left[C_{i}^{n+1}+C_{i+1}^{n+1}\right] \\
& +\frac{K_{i-1 / 2}}{h}\left[\frac{1}{h^{\alpha}} \sum_{j=0}^{i} w_{j}^{\alpha} C_{i-j}^{n+1}\right]-\frac{K_{i+1 / 2}}{h}\left[\frac{1}{h^{\alpha}} \sum_{j=0}^{i+1} w_{j}^{\alpha} C_{i-j+1}^{n+1}\right]
\end{aligned}
$$

Collecting like terms,

$$
\frac{C_{i}^{n+1}-C_{i}^{n}}{\tau}=\frac{1}{h} \sum_{j=0}^{N} g_{i j} C_{j}^{n+1},
$$

where

$$
g_{i j}= \begin{cases}h^{-\alpha} K_{i+1 / 2} w_{i-j+1}^{\alpha}-h^{-\alpha} K_{i-1 / 2} w_{i-j}^{\alpha} & \text { for } j<i-1, \\ h^{-\alpha} K_{i+1 / 2} w_{2}^{\alpha}-h^{-\alpha} K_{i-1 / 2} w_{1}^{\alpha}+V_{i-1 / 2} / 2 & \text { for } j=i-1, \\ h^{-\alpha} K_{i+1 / 2} w_{1}^{\alpha}-h^{-\alpha} K_{i-1 / 2} w_{0}^{\alpha}+\left(V_{i-1 / 2}-V_{i+1 / 2}\right) / 2 & \text { for } j=i \\ h^{-\alpha} K_{i+1 / 2} w_{0}^{\alpha}-V_{i+1 / 2} / 2 & \text { for } j=i+1 \\ 0 & \text { for } j>i+1\end{cases}
$$


For the numerical solution vector $\mathbf{C}^{n}=\left(C_{1}^{n}, \ldots, C_{N-1}^{n}\right)$, we solve the vector equation

$$
\left(\mathrm{I}+\frac{\tau}{\mathrm{h}} \mathrm{A}\right) \mathrm{C}^{\mathrm{n}+1}=\mathrm{C}^{\mathrm{n}},
$$

at each time step, where the matrix $A$ has elements $A_{i j}=-g_{i j}$.

\section{Numerical experiments}

\subsection{Example one}

We compare the finite difference and finite volume methods for a test problem. We consider the space-fractional advection-dispersion equation

$$
\frac{\partial C(x, t)}{\partial t}+\frac{\partial}{\partial x}(V(x) C(x, t))=\frac{\partial}{\partial x}\left[K(x) \frac{\partial^{\alpha} C(x, t)}{\partial x^{\alpha}}\right]
$$

for $(x, t) \in[0,500] \times[0, T]$ with the boundary and initial conditions

$$
\begin{aligned}
& C(0, t)=C(500, t)=0 \quad \text { for } 0 \leqslant t \leqslant T, \\
& C(x, 0)=0.05 \delta(x-25) \quad \text { for } 0 \leqslant x \leqslant 500,
\end{aligned}
$$

and parameters $\mathrm{K}(\mathrm{x})=0.06 \mathrm{x}, \mathrm{V}(\mathrm{x})=0.1 \mathrm{x}$ and $\alpha=0.7$. This scenario was considered by Zhang et al. [12] who used the finite difference method (13) to obtain the numerical solution.

Our numerical solution at time $T=10$ days using $\Delta x=0.5$ and $\tau=0.1$ is shown in Figure 1, and agrees well with that obtained by Zhang et al. [12]. The heavier leading tail and the lagged peak of the fractional model, compared to the standard Gaussian $(\alpha=1)$ model, are clearly visible. The solutions obtained using the finite difference method (13) and finite volume method (29) are visually indistinguishable. The breakthrough curve at $x=300 \mathrm{~m}$ is illustrated in Figure 2 and also agrees with Zhang et al. [12]. The breakthrough 


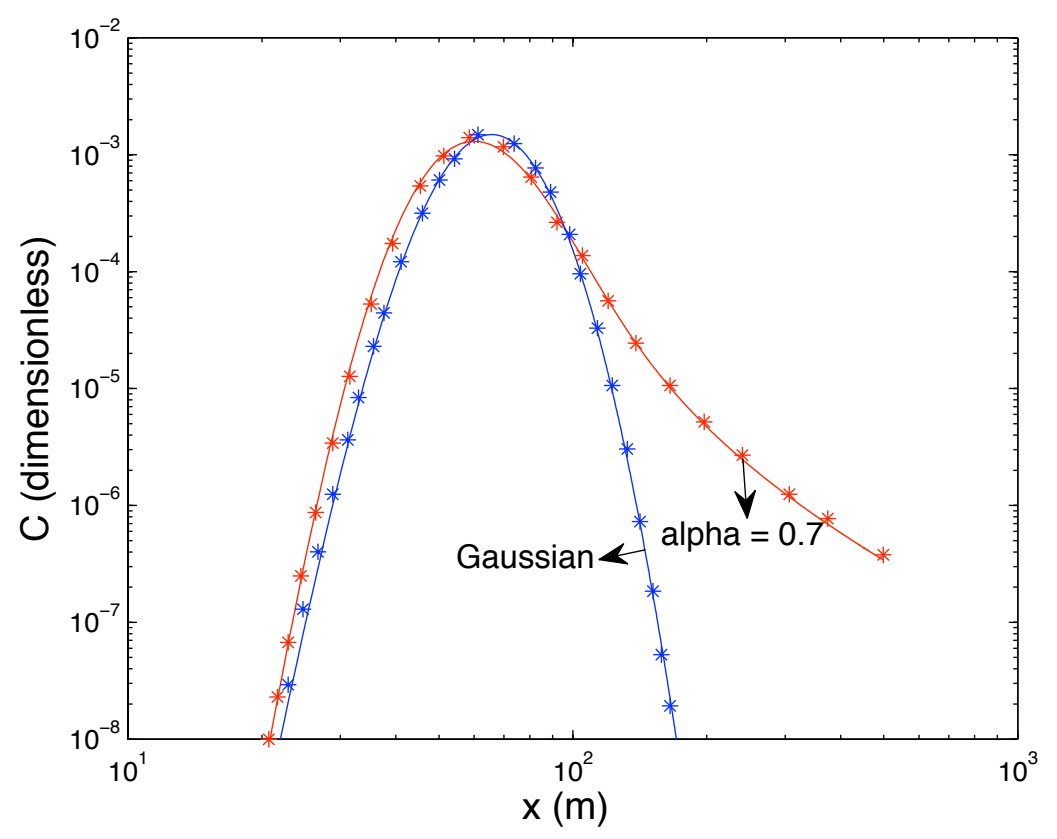

Figure 1: Comparison of our numerical solution (solid lines) and that obtained by digitising Figure 2a by Zhang et al. [12] (symbols) at final time $\mathrm{T}=10$ days using $\Delta x=0.5$ and $\tau=0.1$.

peak of the fractional model arrives slightly behind that of the Gaussian model since the fractional model has more mass when in front of the peak [12]. Analytical solutions are unavailable for this problem. Despite this, we verify numerically that the finite difference and finite volume methods are first order accurate in both space and time. For verification we select sufficiently small values of $h$ and $\tau$, and generate three solutions with successively refined parameters $(h, \tau),(h / 2, \tau / 2)$ and $(h / 4, \tau / 4)$. The order of the method is 


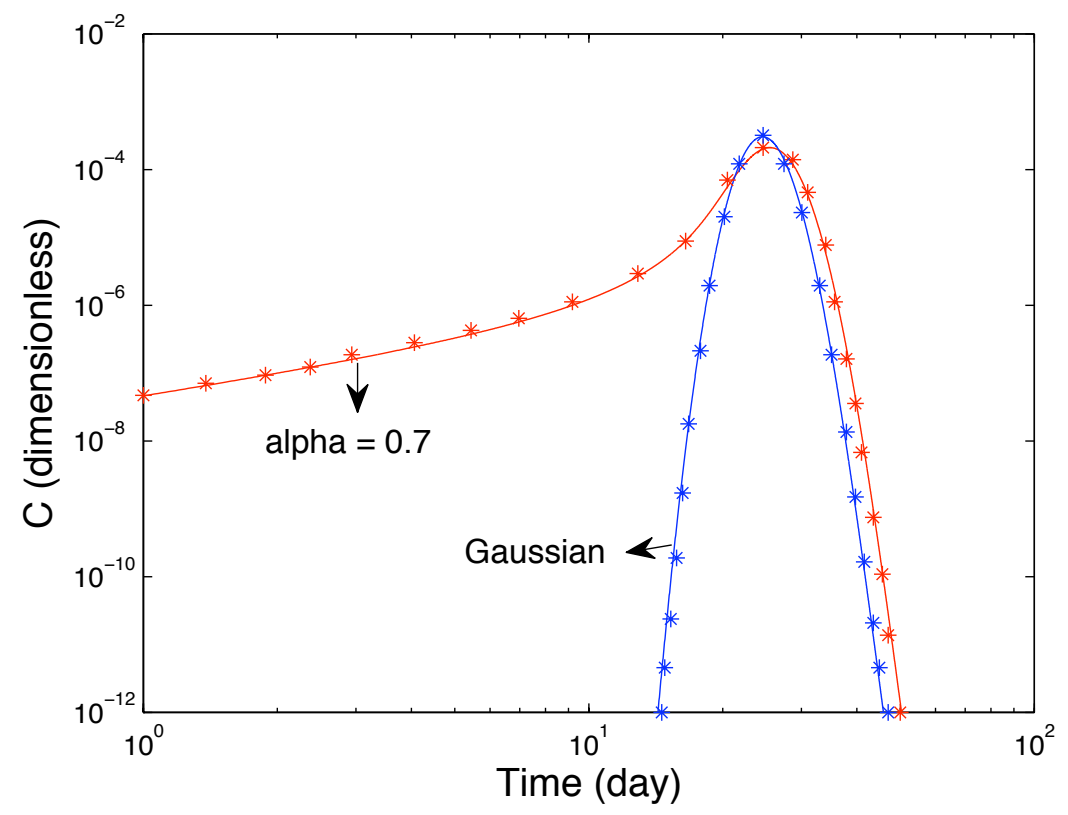

Figure 2: Breakthrough curve of our (solid lines) and that obtained by digitising Figure 3a by Zhang et al. [12] (symbols) at $x=300 \mathrm{~m}$ using $\Delta x=0.5$ and $\tau=0.02$.

then estimated by [4, p.59]

$$
\log _{2}\left(\frac{\max \left|C_{h, \tau}-C_{h / 2, \tau / 2}\right|}{\max \left|C_{h / 2, \tau / 2}-C_{h / 4, \tau / 4}\right|}\right) .
$$

Using $h=0.2$ and $\tau=0.01$, we obtained the value 1.0 for the finite difference method, and 0.98 for the finite volume method, which is consistent with both methods being first order in space and time. 


\subsection{Example two}

We present a test problem to highlight the advantage of the finite volume method compared to the finite difference method by examining the mass balance error for each method. We consider the space-fractional advectiondispersion equation

$$
\frac{\partial C(x, t)}{\partial t}+\frac{\partial}{\partial x}(V(x) C(x, t))=\frac{\partial}{\partial x}\left[K(x) \frac{\partial^{\alpha} C(x, t)}{\partial x^{\alpha}}\right],
$$

for $(x, t) \in[0,500] \times[0, T]$ together with the boundary and initial conditions

$$
\begin{aligned}
& C(0, t)=C(500, t)=0 \text { for } 0 \leqslant t \leqslant T, \\
& C(x, 0)=\delta(x-250) \text { for } 0 \leqslant x \leqslant 500,
\end{aligned}
$$

and parameters $\mathrm{K}(\mathrm{x})=0.06 \mathrm{x}, \mathrm{V}(\mathrm{x})=0.1 \mathrm{x}$ and $\alpha=0.7$.

Although this problem has homogeneous Dirichlet conditions, we still extract meaningful mass balance errors by considering the change in mass in the interior (non-boundary) cells compared to the mass lost due to enforced zero boundary conditions. For the finite volume method this calculation is straightforward as the required fluxes are already computed as part of the discretisation.

For the finite difference method we consider two approaches. The first, method A, computes the required fluxes as an additional post-processing step after obtaining the numerical solution. The second, method B, simply compares the total mass in the finite difference solution, at any time, to that of the finite volume solution.

Figure 3 shows the computed mass balance errors up to final time $T=1$ day with $\Delta x=1$ and $\tau=0.005$. It is readily apparent from the figure that the finite volume method produces a solution that conserves mass (to within machine precision). In contrast, the finite difference solution exhibits mass balance errors many orders of magnitude larger, and growing over time. These 


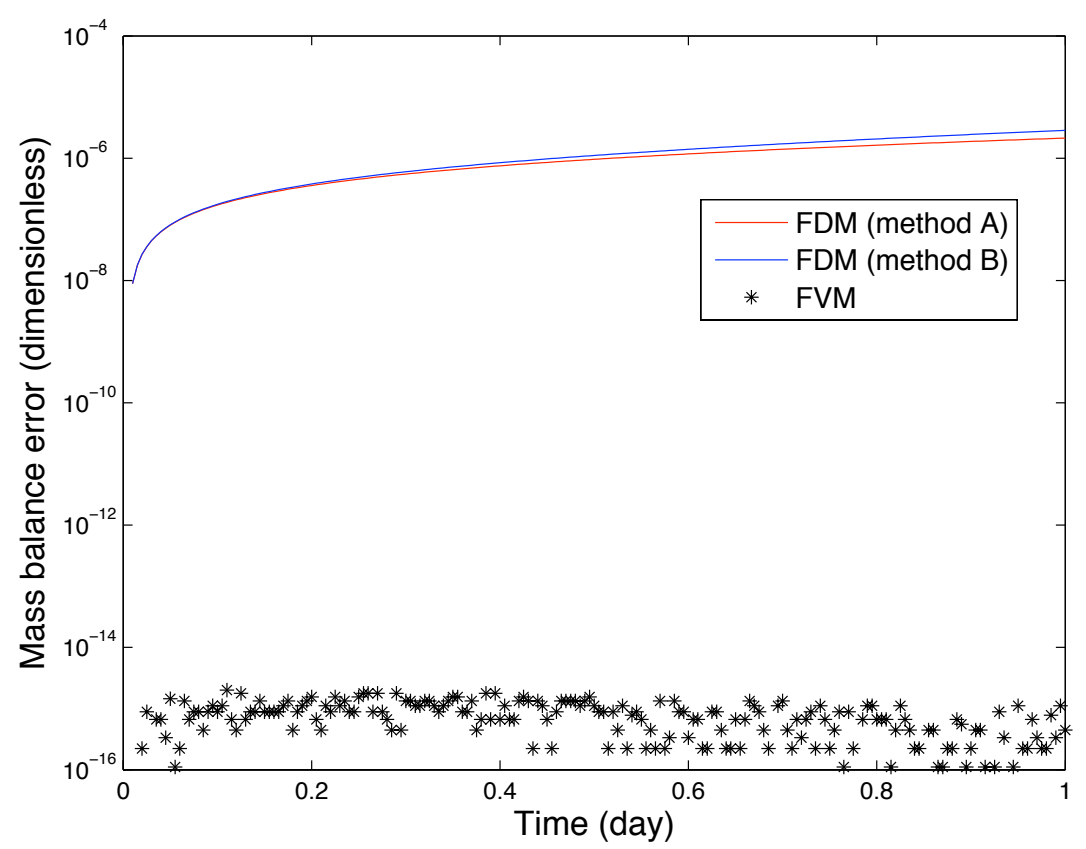

Figure 3: Mass balance error for the finite difference method (FDM), methods $\mathrm{A}$ and $\mathrm{B}$, and the finite volume method (FVM) using $\Delta x=1$ and $\tau=0.005$.

conclusions are independent of whether method A or method B is used to compute the mass balance errors.

This example clearly illustrates the advantage of the finite volume method over the finite difference method for problems of this nature. The inherent conservativeness of the finite volume method yields precise mass balance, whereas the finite difference method provides no such guarantee. 


\section{Conclusion}

We considered a space-fractional advection-dispersion equation with variable coefficients on a one dimensional finite domain with homogeneous Dirichlet boundary conditions. Finite difference methods for solving this equation require that the product rule is first applied, and then the Riemann-Liouville fractional derivatives are discretised using standard and shifted Grünwald formulas, depending on the fractional order. We presented a finite volume method that deals directly with the differential equation in conservative form. Fractionally shifted Grünwald formulas are used to discretise the fractional derivatives at control volume faces, eliminating the need for product rule expansions.

Numerical experiments confirm that both methods recover the correct solution for a test problem. Additionally, both methods are confirmed numerically to be first order in space and time. However, we showed that the finite volume method produces a solution which is conservative, whereas the finite difference method does not. We conclude that the finite volume method is preferable for solving the space-fractional advection-dispersion equation with variable coefficients.

\section{References}

[1] E. E. Adams and L. W. Gelhar. Field study of dispersion in a heterogeneous aquifer: 2. Spatial moments analysis. Water Resources Research, 28:3293-3307 (1992). doi:10.1029/92WR01757 C558

[2] D. A. Benson, S. W. Wheatcraft and M. M. Meerschaert. Application of a fractional advection-dispersion equation. Water Resources Research, 36:1403-1412 (2000). doi:10.1029/2000WR900031 C558 
[3] D. A. Benson, S. W. Wheatcraft and M. M. Meerschaert. The fractional-order governing equation of Lévy motion. Water Resources Research, 36:1413-1423 (2000). doi:10.1029/2000WR900032 C558

[4] J. H. Ferziger and M. Peric. Computational Methods for Fluid Dynamics. Springer-Verlag, Berlin, third edition (2002). C568

[5] H. Hejazi, T. Moroney and F. Liu. Stability and convergence of a finite volume method for the space fractional advection-dispersion equation. Journal of Computational and Applied Mathematics, 255:684-697 (2014). doi:10.1016/j.cam.2013.06.039 C559, C564, C565

[6] M. M. Meerschaert and C. Tadjeran. Finite difference approximations for fractional advection-dispersion flow equations. Journal of Computational and Applied Mathematics, 172:65-77 (2004). doi:10.1016/j.cam.2004.01.033 C559, C560, C561

[7] M. M. Meerschaert and C. Tadjeran. Finite difference approximations for two-sided space-fractional partial differential equations. Applied Numerical Mathematics, 56:80-90 (2006). doi:10.1016/j.apnum.2005.02.008 C559

[8] K. W. Morton. Numerical solution of convection-diffusion problems. Springer, London, first edition (1996). C561

[9] I. Podlubny. Fractional Differential Equations. Academic Press, New York (1999). C560

[10] C. Tadjeran, M. M. Meerschaert and H. P. Scheffler. A second-order accurate numerical approximation for the fractional diffusion equation. Journal of Computational Physics, 213:205-213 (2006). doi:10.1016/j.jcp.2005.08.008 C559

[11] X. Zhang, J. W. Crawford, L. K. Deeks, M. I. Stutter, A. G. Bengough and I. M. Young. A mass balance based numerical method for the fractional advection-dispersion equation: Theory and application. 
Water Resources Research, 41:W07029 (2005).

doi:10.1029/2004WR003818 C559

[12] Y. Zhang, D. A. Benson, M. M. Meerschaert and E. M. LaBolle. Space-fractional advection-dispersion equations with variable parameters: Diverse formulas, numerical solutions, and application to the Macrodispersion Experiment site data. Water Resources Research, 43:W05439 (2007). doi:10.1029/2006WR004912 C558, C562, C566, C567, C568

[13] Y. Zhang, D. A. Benson and D. M. Reeves. Time and space nonlocalities underlying fractional-derivative models: Distinction and literature review of field applications. Advances in Water Resources, 32:561-581 (2009). doi:10.1016/j.advwatres.2009.01.008 C558

\section{Author addresses}

1. H. Hejazi, School of Mathematical Sciences, Queensland University of Technology, GPO Box 2434, Brisbane, QLD 4001, Australia. mailto:hala.hejazi@student.qut.edu.au

2. T. Moroney, School of Mathematical Sciences, Queensland University of Technology, GPO Box 2434, Brisbane, QLD 4001, Australia. mailto:t.moroney@qut.edu.au

3. F. Liu, School of Mathematical Sciences, Queensland University of Technology, GPO Box 2434, Brisbane, QLD 4001, Australia. mailto:f.liu@qut.edu.au 